\title{
Leiomyosarcoma of the ovary associated with leiomyoma of uterus: a case report
}

\section{Chitra Thyagaraju*, Jayalakshmi Durairaj, Sujatha Venkataraman}

\author{
Department of Obstetrics \& Gynaecology, Jawaharlal Institute of Post-graduate Medical Education and Research, \\ Pondicherry, India
}

Received: 27 April 2015

Accepted: 10 May 2015

\author{
*Correspondence: \\ Dr. Chitra Thyagaraju, \\ E-mail: drchitra@yahoo.com
}

Copyright: () the author(s), publisher and licensee Medip Academy. This is an open-access article distributed under the terms of the Creative Commons Attribution Non-Commercial License, which permits unrestricted non-commercial use, distribution, and reproduction in any medium, provided the original work is properly cited.

\begin{abstract}
Leiomyosarcoma of the ovary is a rare disease accounting for $<1 \%$ of all sarcomas. It occurs mainly in postmenopausal women with aggressive behaviour and poor prognosis. Surgery is the cornerstone of treatment, while the role of chemotherapy and radiotherapy is still not clear. This paper presents the case of a post-menopausal woman who is diagnosed with primary leiomyosarcoma of the ovary along with multiple leiomyoma of the uterus, and who receives a surgical approach and currently achieving 25 months free from disease.
\end{abstract}

Keywords: Leiomyosarcoma of ovary

\section{INTRODUCTION}

Primary sarcomas comprises $3 \%$ of all ovarian malignancies and leiomyosarcoma of the ovary accounts for $<1 \%$. They are usually seen in postmenopausal women, have a poorer prognosis due to their late presentation and aggressive nature. Because of its extreme rarity and its first time presentation along with multiple leiomyoma of the uterus in the literature, we present this case of ovarian neoplasm in a postmenopausal woman.

\section{CASE REPORT}

A 55 years old multiparous, post-menopausal woman presented with history of pain abdomen and abdominal distension for 5months duration. Her past medical and obstetric history was uneventful. She was thin built, poorly nourished and anaemic on general examination. On abdomen examination, a 36 weeks irregular mass which was firm to hard in consistency with restricted mobility was felt without sign of ascites. The lower margin of mass was made out. On per speculum examination, cervix was deviated to left side. On pelvic examination, uterus was not felt separately and mass was felt through all fornixes without any nodule in POD.

Her haemoglobin was $7.6 \mathrm{gm}$ and peripheral smear was suggestive of iron deficiency anaemia. Other haematological, hepatic and renal parameters were normal. Tumour markers CA-125 and CEA were within normal limits. Ultrasound and CT confirmed a mixed heterogeneous mass with both solid and cystic (28x20x15 $\mathrm{cm})$ components with few calcific areas and few enhancing thin septae. No free fluid abdomen or pleural effusion.

Provisional diagnosis of Malignant Ovarian Tumour was made and patient was posted for Staging Laparotomy. Intra-operatively, huge heterogeneous ovarian mass measuring approximately $30 \times 30 \mathrm{~cm}$ with solid and cystic areas with few necrotic areas were noted. Uterus was enlarged to 14 weeks with multiple fibroids largest measuring 7x6 cm broad ligament fibroid on left side. Left ovary was normal. On thorough exploration, no metastatic deposits seen in pelvic cavity and other 
visceral organs. Total Abdominal Hysterectomy with excision of the ovarian tumour along with bilateral salpingo-oophorectomy with partial omentectomy done. Post op period was uneventful. She did not receive any postoperative adjuvant therapy. Follow-up 21 months after initial surgery, showed no evidence of recurrence and the patient is doing fine.

Histopathology showed the features of leiomyosarcoma of ovary. Tumour was composed of fascicles of spindle cells in interlacing bundles with diffuse mild to moderate pleomorphic, mitosis 12/10 HPF and areas of coagulative necrosis. These cells are positive for SMA and vimentin.

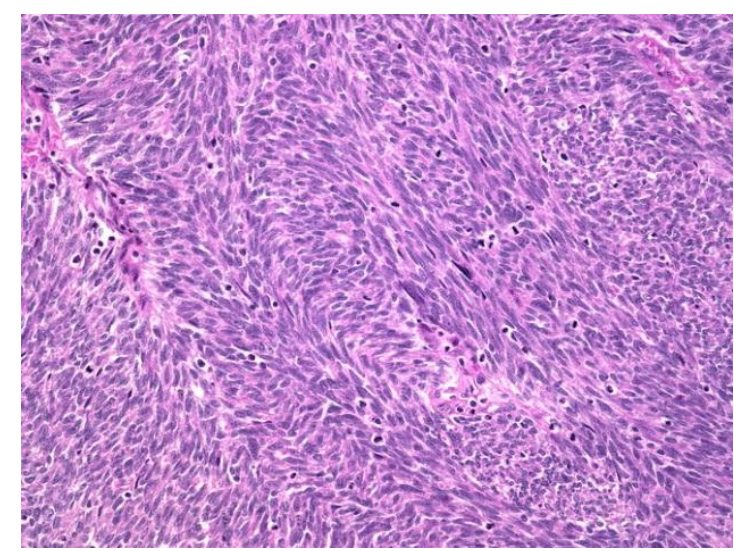

Figure 1: The tumor showed moderate cytologic atypia and >20 mitotic figures/10 HPF with areas of coagulative necrosis.

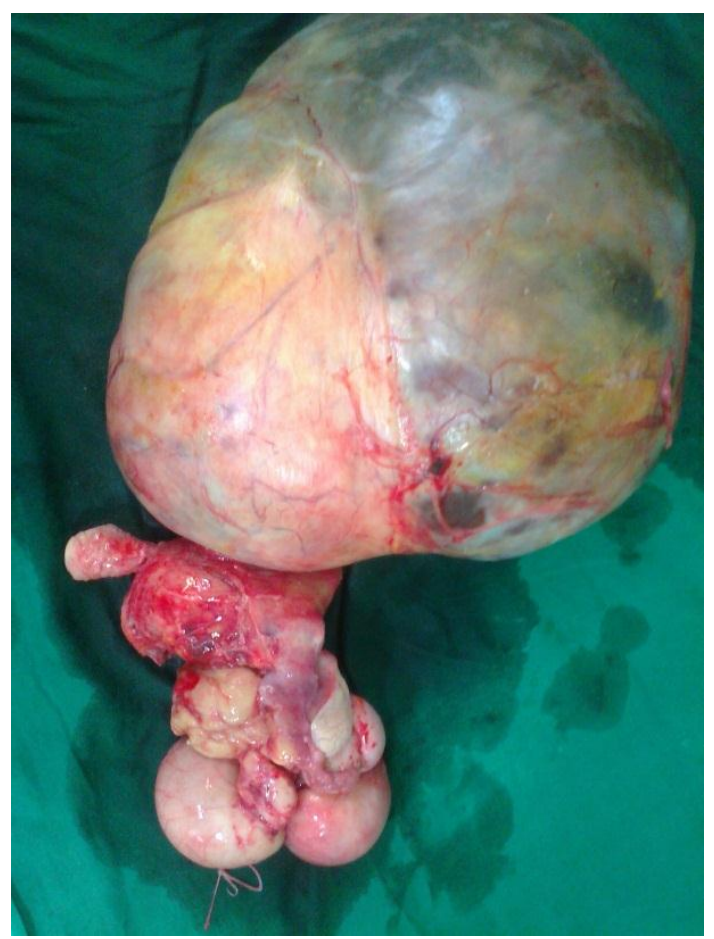

Figure 2: Tumour showing solid with cystic areas containing haemorrhagic fluid and multiple firbroid uterus.

\section{DISCUSSION}

Leiomyosarcoma of the ovary accounts for 7\%-8\% of all soft tissue sarcomas, other rare sites of leiomyosarcoma are stomach, vagina, bone, small intestine. ${ }^{1}$ Primary ovarian sarcomas comprises heterogeneous group of tumours, representing $3 \%$ of all ovarian neoplasm, affecting mainly post-menopausal women. ${ }^{2,3}$ However some cases have been reported in adolescents. There is no case reported in literature the presentation of leimyosarcoma of ovary with leiomyoma of the uterus.

The pathogenesis is uncertain. They originate from hilar smooth muscles, smooth muscles of blood vessels, totipotent stem cells of ovary or metaplasia of ovarian stroma. ${ }^{4,5}$ The tumours are usually asymptomatic or present with non-specific symptoms and signs.

Macroscopically, these tumours are large, unilateral, and solid with haemorrhagic and cystic degeneration. ${ }^{6}$ Microscopically, tumours varies from well-differentiated to highly pleomorphic sarcoma. Presence of moderate to severe cytological atypia with more than 10 mitosis/10 HPF with features of tumour cell necrosis suggestive of malignancy. ${ }^{4}$ They have reactivity positive for dermin, vimentin, and actin and negative for cytokeratin, CD34 and S100. ${ }^{5,7}$

The differential diagnosis of OLS include fibro sarcomas, spindle cell sarcoma, Krukenberg tumour, mixed Mullerian tumour, undifferentiated carcinoma, GIST Gastro-intestinal stromal tumour. ${ }^{4,8}$ The classification of ovarian tumours from IFG\&O is recommended for staging purpose of these tumours. ${ }^{4,5}$

Radical surgery is the treatment of choice for OLS followed by adjuvant chemotherapy or radiotherapy. In most of these patients the disease confers a poorer prognosis with majority dying within 1 year of diagnosis. $^{7}$

Adjuvant chemotherapy are of unproven benefit, especially in disease confined to the ovaries, as with our patient who is disease free for 15 months, which is similar to the case reported by Kumar et al., ${ }^{9}$ where the mass was explored and resected and on follow up, patient was disease free for 2 years.

Rasmussen et al described the usefulness of performing cytoreduction surgery in repeated opportunities during relapses and they also suggested that primary leiomyosarcomas of the ovary could be hormonesensitive. In tumours confined to the ovary some authors have described that surgery could be enough as sole treatment. ${ }^{3}$

But in cases of large ovarian tumour, to avoid metastasis, adjuvant chemotherapy and radiotherapy is necessary to increase survival in these patients and in majority of these patients radical surgery itself will be difficult. 


\section{CONCLUSION}

In this study, we presented a case of ovarian leiomyosarcoma with multiple fibroid uterus with normal serum tumour marker level. We found that even in advanced cases, surgical debulking gives good symptomatic relief, so appropriate surgical treatment should not be delayed solely on the basis of normal tumor marker levels. Because of its poor prognosis, early diagnosis and prompt surgical removal are important for patients with ovarian leiomyosarcoma for improvement of symptoms and in survival.

Funding: No funding sources Conflict of interest: None declared

Ethical approval: Not required

\section{REFERENCES}

1. Enzinger FM, Weiss SW. Soft tissue sarcoma. The CV Mosby Co. 1988;2:402.

2. Kurian RR, Preethi J, Remadevi AV. Leiomyosarcoma of ovary: a case report. Indian $\mathrm{J}$ Pathol Microbiol. 2005;48(1):19-20.

3. Rasmussen CC, Skilling JS, Sorosky JI, Lager DJ, Buller RE. Stage IIIC ovarian leiomyosarcoma in a premenopausal woman with multiple recurrences: prolonged survival with surgical therapy. Gynecol Oncol. 1997;66(3):519-25.

4. Bouie SM, Cracchiolo B, and Heller D. Epithelioid leiomyosarcoma of the ovary. Gynecol Oncol. 2005;97:697-9.

5. Taşkin S, Taşkin EA, Uzüm N, Ataoğlu O, Ortaç F. Primary ovarian leiomyosarcoma: a review of the clinical and immunohistochemical features of the rare tumor. Obstet Gynecol Surv. 2007;62:480-6.

6. Prat J, Fox H. Mesenchymal tumour of the ovary. Obstet Gynaecol. 1987;2(2):701-2.

7. Dixit S, Singhal S, Baboo HA, Vyas RK, Neema JP, Murthy R, et al. Leiomyosarcoma of the ovary - a case report. J Postgrad Med. 1993;39(3):151-3.

8. Arslan OS, Sumer C, Cihangiroglu G, Kanat-Pektas M, Gungor T. A rare tumor of the female genital tract: primary ovarian leiomyosarcoma. Arch Gynecol Obstet. 2011;283:83-5.

9. Kumar RG. Leiomyosarcoma of probably ovarian or broad ligament origin. $\mathrm{Br} \mathrm{J}$ Obstet Gynecol. 1982;88:327-9.

DOI: $10.18203 / 2320-1770 . i j r \operatorname{cog} 20150103$

Cite this article as: Thyagaraju C, Durairaj J, Venkataraman S. Leiomyosarcoma of the ovary associated with leiomyoma of uterus: a case report. Int J Reprod Contracept Obstet Gynecol 2015;4:837-9. 